\title{
Composição e riqueza de Odonata (Insecta) em riachos com diferentes níveis de conservação em um ecótone Cerrado-Floresta Amazônica
}

\author{
Leandro JUEN ${ }^{1 *}$, José Max Barbosa de OLIVEIRA-JUNIOR², Yulie SHIMANO² \\ Thiago Pereira MENDES 3 , Helena Soares Ramos CABETTE ${ }^{4}$ \\ 1 Universidade Federal do Pará-UFPA. Laboratório de Ecologia Aquática, Instituto de Ciências Biológicas. Rua Augusto Correia, nº 1, Bairro Guamá, CEP: 66075-110. Belém, Pará,

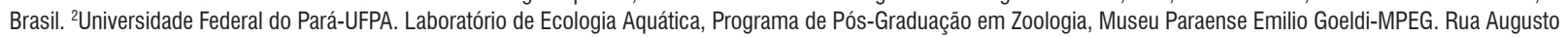 \\ Correia, nº 1, Bairro Guamá, CEP: 66075-110. Belém, Pará, Brasil. josemaxoliveira@gmail.com, shimano.yulie@gmail.com \\ ${ }^{3}$ Universidade Federal do Pará-UFPA. Laboratório de Ecologia Aquática, Programa de Pós-Graduação em Ecologia Aquática e Pesca. Rua Augusto Correia, nº 1, Bairro Guamá, \\ CEP: 66075-110. Belém, Pará, Brasil. thiagomendes.bio@gmail.com \\ 4 Universidade do Estado de Mato Grosso-UNEMAT. Laboratório de Entomologia, Departamento de Biologia. Cx.P.08, CEP: 78690-000. Nova Xavantina, Mato Grosso, Brasil. \\ hcabette@uol.com.br \\ * Autor Correspondente: leandrojuen@ufpa.br
}

\section{RESUMO}

A retirada ou a substituição da vegetação ripária provoca uma alteração no ambiente físico, no fluxo sazonal e na qualidade química da água. Essas modificaçôes podem causar a diminuiçáo da riqueza pela extinção local de espécies. O objetivo deste trabalho foi avaliar o efeito do distúrbio da integridade ambiental sobre a riqueza e composição de espécies de Odonata adultos em córregos com diferentes níveis de conservação, na Bacia do Rio Suiá-Missu, Mato Grosso, Brasil. As modificaçóes nos sistemas aquáticos afetaram a comunidade de Odonata, provavelmente devido às exigências ecofisiológicas e comportamentais relacionadas a adultos e larvas. Anisoptera, que necessitam de ambientes com maior incidência de sol devido ao tamanho do corpo, apresentaram maior riqueza de espécies em ambientes com menor cobertura vegetal. Por outro lado, os Zygoptera geralmente habitam riachos com cobertura vegetal mais densa, e por isso, apresentaram um decréscimo de sua riqueza em locais alterados, devido à maior entrada de luz e/ou variaçáo do calor. Assim, para as duas subordens, as alteraçóes ambientais não precisam ser severas para produzir modificaçóes significativas na composição, indicando que os serviços ecossistêmicos poderiam ser perdidos, mesmo com alteraçóes parciais do meio físico.

PALAVRAS-CHAVE: ambientes aquáticos; biodiversidade; integridade ambiental; libélulas.

\section{Composition and richness of Odonata (Insecta) in streams with different levels of conservation in a Cerrado-Amazonian Forest ecotone}

\begin{abstract}
The removal or substitution of riparian vegetation causes disturbance in physical environment, seasonal water flow and water chemical quality. These modifications can cause decrease in species richness by local extinctions. The aim of this study was to examine the effect of disturbance in the physical environmental on the richness and species composition of Odonata adults in streams with different levels of conservation in the river Suiá-Missu basin, Mato Grosso, Brazil. Modifications in the aquatic systems affected the Odonata community, probably because their ecophysiological and behavioral requirements of adults and larvae. Anisoptera species, which require sunny environments because of their body size, had higher species richness in environments with low plant cover. On the other hand, Zygoptera species, which generally inhabit streams with dense vegetation, presented a decrease in richness in disturbed environments, as a result high sunlight radiation and/or variations in temperature. Hence, in both suborders, environmental perturbations do not need to be severe to change species composition, indicating that ecosystem services could be lost, even with only partial alterations in physical environment.
\end{abstract}

KEYWORDS: aquatic environments; biodiversity; environmental integrity; dragonflies. 


\section{INTRODUÇÃO}

Alteraçôes na paisagem de um ambiente exercem grande influência na riqueza de espécies (Callisto et al. 2001), porque diminuem a disponibilidade de recursos criando manchas de hábitats que tem sua recolonização dependente da migraçáo de espécies provenientes de outros locais mais adequados. Em rios de regiáo temperada, o desmatamento tem provocado alteraçóes nas assembleias de macroinvertebrados, devido às mudanças na entrada de luz, variação de temperatura, níveis de nutrientes, tamanho das partículas do substrato, distribuição e disponibilidade dos recursos alimentares afetando drasticamente a integridade dos ambientes aquáticos (Bojsen e Jacobsen 2003). Padrôes similares de modificação na estrutura da comunidade tem sido detectado na regiáo tropical (Dias-Silva et al. 2010; Pereira et al. 2012; Silva-Pinto et al. 2012) incluindo regiáo Amazônica (Monteiro-Júnior et al. 2013; Oliveira-Júnior et al. 2013).

Quanto maior for à especialização e os requisitos ambientais de uma espécie, espera-se maior variação na abundância e no risco de extinção local frente a alteraçôes antrópicas, como já detectado para a ordem Odonata (Monteiro-Júnior et al. 2013; Carvalho et al. 2013). Os representantes dessa ordem habitam todos os tipos de ambientes de água doce, e a composição de espécies pode variar de acordo com as alteraçôes das variáveis ambientais, entre as quais, a presença de vegetação marginal (Silva et al. 2010; Guillermo-Ferreira e Del-Claro 2012; Monteiro-Júnior et al. 2013) ou de macrófitas (Juen et al. 2007; Alves-Martins et al. 2012), hidroperíodo, concentração de poluentes, condutividade, $\mathrm{pH}$, correnteza, largura, profundidade (Juen e De Marco 2011), oxigênio dissolvido, temperatura e vazão (Corbet 1999). Isto se dá por que há uma grande variação das exigências ecofisiológicas dentro da ordem, o que nos permitem fazer prediçôes e testar hipóteses distintas entre as subordens (para maiores informaçôes ver Corbet 1999).

Anisoptera e Zygoptera apresentam distribuição influenciada pelo clima, fatores físico-químicos do gradiente e integridade ambiental (Juen e De Marco 2012), mas Zygoptera demonstra maiores requisitos ambientais, por apresentar comportamento perchers (ficam em poleiros defendendo melhores áreas para efetuar a cópula e oviposição, bem como para melhor visualização das fêmeas), ou seja, capacidade dispersiva mais restrita com alta dependência da estrutura do hábitat (Corbet 1999; Heckman 2008; Heiser e Schmitt 2010). Ainda, os integrantes dessa subordem habitam, geralmente, riachos com cobertura vegetal densa, e por isso, espera-se que eles apresentem maior riqueza de espécies em ambientes preservados e um decréscimo de sua riqueza em locais alterados, pois náo conseguiriam sobreviver em ambientes com grande entrada de luz e calor (Carvalho et al. 2013). Já as espécies de Anisoptera, são ditos voadores (fliers), e sendo maiores, necessitam de áreas com maior incidência de sol (Corbet 1999), desta forma, a riqueza de espécies desse grupo deve ser maior em ambientes com menor cobertura vegetal.

Nesse estudo testamos as hipóteses de que: 1) a composição de Odonata estará modificada em córregos alterados e degradados quando comparada com locais de alta integridade; 2) a riqueza de Anisoptera terá acréscimo de espécies em córregos alterados e degradados; 3 ) e que a riqueza de espécies de Zygoptera será maior em ambientes preservados. O objetivo deste trabalho foi avaliar o efeito do distúrbio da integridade de córregos e rios com diferentes níveis de conservação sobre a riqueza e a composição de espécies de adultos de Odonata, na Bacia do Rio Suiá-Missu, MT, Brasil.

\section{MATERIAL E MÉTODOS}

\section{Área de estudo}

Localizada na região centro-leste do estado de Mato Grosso, aproximadamente entre as coordenadas $11^{\circ} 15^{\prime} \mathrm{e}$ $13^{\circ} 30^{\prime}$ S e $51^{\circ} 30^{\prime}$ e $53^{\circ} 15^{\prime}$ W (Maeda et al. 2008), a Bacia Hidrográfica do Rio Suiá-Missu está situada em uma área de transição entre Cerrado e Floresta Amazônica (Figura 1). A regiấo apresenta clima tropical sazonal com uma estaçáo seca (de maio a outubro) e uma chuvosa (de novembro a abril). O clima da região é predominantemente do subtipo Savana (Aw) com microrregiôes características do Subtipo Monçôes (Am) e clima Tropical Chuvoso (A), segundo a classificação de Köppen. Com precipitação média anual de cerca de 1.370 $\mathrm{mm}$ e temperatura máxima de $32,7^{\circ} \mathrm{C}$ e mínima de $17,0^{\circ} \mathrm{C}$ (Ratter et al. 1978).

A Bacia abriga trechos ainda preservados de tipos vegetacionais distintos, e que, segundo Ratter et al. (1978), merecem destaques as fisionomias de cerrado sensu stricto, as áreas de Mata Seca e as de cerrado transicional denominado "cerradão de Hirtella glandulosa" em função da espécie de maior IVI (Importance Value Index). Os principais usos do solo são para a plantação de soja ou de pastagem para a criação de gado.

\section{Coleta de dados}

Foram amostrados 12 córregos da Bacia do Suiá-Missu, de primeira a sexta ordens, de acordo com a classificação de Strahler (1957). Seis córregos localizam-se em áreas de cerradão, três em áreas de cerrado sensu stricto e três em área de mata (Figura 1, Tabela 1). As amostragens foram realizadas em três períodos do ano: seca (setembro/2007), início das chuvas (dezembro/2007) e vazante (março/2008).

O Índice de Integridade de Hábitat (Habitat Integrity Index-HII) (Nessimian et al. 2008) foi utilizado para avaliar a integridade dos pontos amostrados. Este protocolo é 
Tabela 1. Locais, siglas, coordenadas e fitofisionomias dos pontos de coleta amostrados na Bacia Hidrográfica do Rio Suiá-Missu, MT, Brasil.

\begin{tabular}{|c|c|c|c|}
\hline Local & Siglas & Coordenadas geográficas & Fitofisionomic \\
\hline Córrego Brejão & CRBJ & $12^{\circ} 38^{\prime} 32,3^{\prime \prime}$ S, 515'20,6” W & Cerradão \\
\hline Córrego Lúcio & CRL & $13^{\circ} 05^{\prime} 34,5^{\prime \prime} \mathrm{S}, 52^{\circ} 15^{\prime} 16,9^{\prime \prime} \mathrm{W}$ & Cerradão \\
\hline Córrego Sucuri & CRSRI & $11^{\circ} 49^{\prime} 50,7^{\prime \prime}$ S, 52¹7’02,2” W & Mata Seca \\
\hline Córrego Transição-brejo & CRTB & $13^{\circ} 03^{\prime} 35,6^{\prime \prime}$ S, 52¹2’03,3" W & Cerradão \\
\hline Rio Betis local 1 & RIBET1 & $12^{\circ} 22^{\prime} 28,7^{\prime \prime}$ S, 52॰13'23,1" W & Cerradão \\
\hline Rio Betis local 2 & RIBET2 & $12^{\circ} 22^{\prime} 27,5^{\prime \prime}$ S, 52¹3'19,0” W & Cerradão \\
\hline Rio Darro & RID & $12^{\circ} 21^{\prime} 12,3^{\prime \prime}$ S, 52²1'27,4” W & Mata Seca \\
\hline Rio Piabanha & RIPB & $13^{\circ} 15^{\prime} 34,4^{\prime \prime}$ S, 5209’00,5” W & Cerrado s.s. \\
\hline Rio Suiá-Missu local 1 & RISU1 & $13^{\circ} 15^{\prime} 45,5^{\prime \prime} \mathrm{S}, 52^{\circ} 02^{\prime} 50,9^{\prime \prime} \mathrm{W}$ & Cerrado s.s. \\
\hline Rio Suiá-Missu local 2 & RISU2 & $13^{\circ} 15^{\prime} 24,3^{\prime \prime} \mathrm{S}, 52^{\circ} 08^{\prime} 44,5^{\prime \prime} \mathrm{W}$ & Cerrado s.s. \\
\hline Rio Suiá-Missu local 3 & RISU3 & $11^{\circ} 50^{\prime c 17,8 ”}$ S, 52॰15’07,5” W & Mata Seca \\
\hline Rio Suiazinho & RISUZ & $12^{\circ} 38^{\prime} 33,4^{\prime \prime}$ S, 5256’50,7” W & Cerradão \\
\hline
\end{tabular}

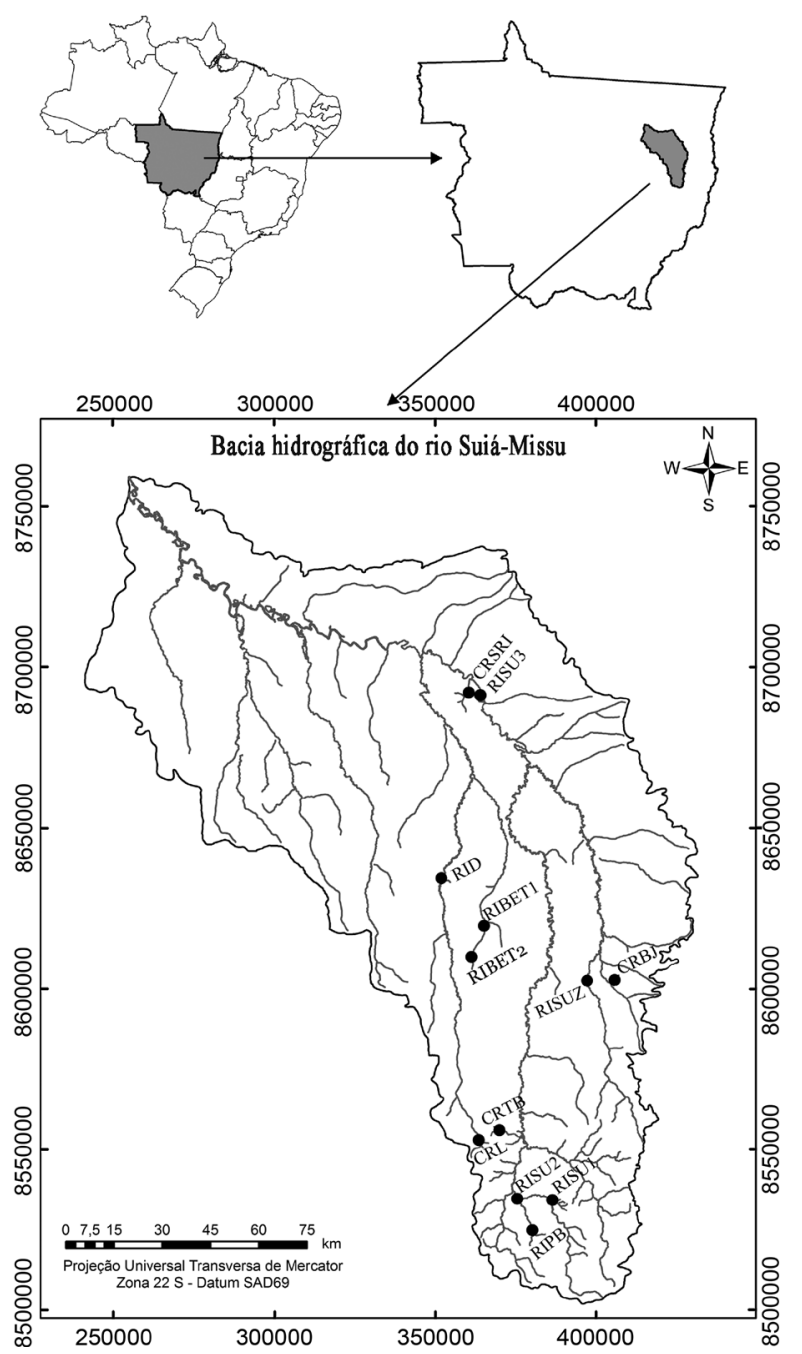

Figura 1- Pontos de coleta de Odonata adultos na Bacia do Rio Suiá-Missu, MT, Brasil, 2007/2008: Rio Suiá-Missu local 1 (RISU1), Rio Suiá-Missu local 2 (RISU2), Rio Suiá-Missu local 3 (RISU3), Rio Betis local 1 (RIBET1), Rio Betis local 2 (RIBET2), Rio Piabanha (RIPB), Rio Darro (RID), Rio Suiazinho (RISUZ), Córrego Brejão (CRBJ), Córrego Transição-brejo (CRTB), Córrego Sucuri (CRSRI) e Córrego Lúcio (CRL). constituído por doze itens que descrevem as condiçōes ambientais locais. Cada item é composto por quatro a seis alternativas ordenadas de forma a representar sistemas cada vez mais íntegros. O valor do índice varia de zero a um, indicando um gradiente crescente de integridade. O HII avaliado para os córregos variou entre 0,39 a 0,74 .

Classificar um ambiente quanto a seu estágio de conservação ou características físicas é sempre difícil e muitas vezes subjetivo. Para resolver esse problema, foi estabelecida a classificação utilizando as 12 questôes do HII, que considera as condiçôes de alteraçáo do ambiente, e também a largura e profundidade do córrego. A largura foi utilizada, pois acreditamos que córregos largos, por mais conservados que sejam, tem uma comunidade diferente dos córregos estreitos devido a maior entrada de luz no sistema, da mesma forma que a profundidade pode afetar na composição das comunidades. Com esse conjunto de informaçóes foi realizada uma Análise de Escalonamento Multidimensional NãoMétrico (NMDS) para ordenar os locais com base nas suas similaridades ambientais, na qual a medida de dissimilaridade utilizada foi a distância euclidiana. Toda a análise seguiu as recomendações e instruçôes contidas em Legendre e Legendre (1998). Classificar as categorias ambientais considerando essas métricas pode evitar tendenciosidade dos resultados e auxilia na redução da multidimensionalidade dos dados. Sendo assim, com base no agrupamento encontrado na ordenação (Figura 2), os córregos CRTB, RIBET1 e RIBET2 foram classificados aqui como degradados, os córregos e rios CRSRI, RID, CRBJ e RISU3 foram classificados em alterados e RISU1, RISU2, RISUZ, RIPB e CRL em preservados.

As amostragens dos adultos de Odonata foram realizadas seguindo a metodologia de varredura em áreas fixas, já utilizadas com sucesso em outros trabalhos (e. g. Juen e De Marco 2011; Silva-Pinto et al. 2012; Oliveira-Júnior et al. 2013). Com o uso de rede entomológica foram coletados os indivíduos presentes em 100 metros do corpo d'água de cada área, divididos em 20 segmentos de cinco metros. O tempo 


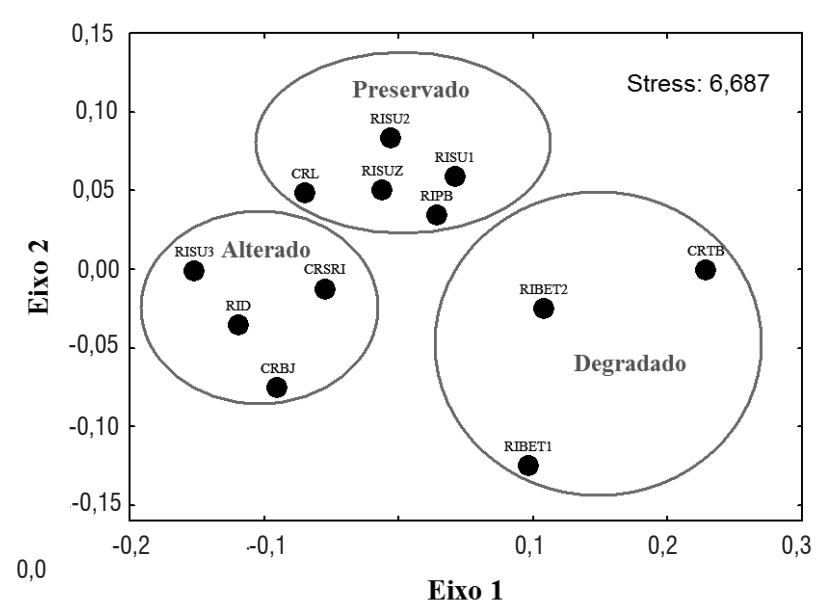

Figura 2. -Ordenação de escalonamento multidimensional não-métrico (NMDS) dos córregos amostrados na Bacia do Rio Suiá-Missu, MT, Brasil, com base nas características físicas e níveis de conservação dos mesmos. Os pontos RISU1, RISU2, CRL, RISUZ e RIPB foram considerados preservados, RISU3, CRSRI, RID e CRBJ foram considerados alterados e CRTB, RIBET1 e RIBET2 foram considerados degradados.

médio de permanência em cada ponto de amostragem foi de uma hora. Concomitantemente, foi mensurada a temperatura do ar em local sombreado perto dos corpos d'água. As temperaturas variaram, entre 29 a $40{ }^{\circ} \mathrm{C}$ em setembro, entre 26 a $38^{\circ} \mathrm{C}$ em dezembro, e 24 a $32^{\circ} \mathrm{C}$ em março.

Para que as coletas fossem realizadas, era necessariamente observada a incidência dos raios solares, que deveriam estar alcançando o canal dos córregos, visando garantir as condiçóes mínimas necessárias para que todos os grupos ecofisiológicos de Odonata (conformadores, heliotérmicos e endotérmicos) estivessem ativos (May 1976; De Marco e Resende 2002). Sendo assim, as amostragens tiveram início entre $10 \mathrm{~h}: 30 \mathrm{~min}$ e $14 \mathrm{~h}: 30 \mathrm{~min}$. Os indivíduos coletados foram acondicionados em envelopes de papel e imersos em acetona P.A. (Puro para Análise), durante 12 horas para Zygoptera e 48 a 72 horas para Anisoptera, para fixação (Lencioni 2005; 2006). Após a secagem por evaporação, os mesmos foram acondicionados em envelopes plásticos sobre papel cartấo e posteriormente depositados como material testemunho na coleção Zoobotânica "James Alexander Ratter" da Universidade do Estado de Mato Grosso, campus de Nova Xavantina, Brasil.

Para identificação das espécies, foram utilizadas chaves taxonômicas especializadas (Garrison et al. 2006; Garrison et al. 2010; Lencioni 2005; 2006), além de comparação com indivíduos da referida coleção.

\section{Análise dos dados}

Os 20 segmentos em cada córrego não são considerados amostras independentes, mas sim pseudorréplicas dentro do mesmo córrego, e por isso, foram usados apenas para estimar a riqueza de espécies de cada córrego. Para as outras análises, a amostra é o córrego (somatório das abundâncias das espécies nos 100 metros). Como nossa pergunta é espacial e não temporal, as amostragens em cada córrego durante as três campanhas foram agrupadas, totalizando 12 amostras.

A medida de riqueza de espécies foi realizada através do estimador não paramétrico jackknife de primeira ordem (Colwell e Coddington 1994) sempre controlando o esforço de amostragem. A técnica de estimativa foi realizada no programa EstimateS (Statistical Estimation of Species Richness and Shared Species from Samples, versão 7.5) (Colwell 2005).

Para comparar a composição de espécies entre os ambientes lóticos foi usada a análise de Escalonamento Multidimensional Não-Métrico (NMDS). A medida de dissimilaridade utilizada nessa análise foi o Índice de Bray-Curtis (Legendre e Legendre 1998). Para testar o grau de conservação dos riachos utilizamos ANOSIM (Clarke e Warwick 1994). As análises (NMDS e ANOSIM) foram feitas no programa $R$ usado os pacotes Vegan e MASS (R Development Core Team 2011).

\section{RESULTADOS}

\section{Descrição da comunidade de Odonata}

Foram coletados 2.233 espécimes de Odonata, distribuídos em oito famílias, 42 gêneros e 79 espécies. A subordem Zygoptera contribuiu com 1.744 indivíduos, distribuídos em seis famílias (Coenagrionidae, Polythoridae, Protoneuridae, Megapodagrionidae, Calopterygidae, Dicterididae), 19 gêneros e 40 espécies. Anisoptera contribuiu com 489 indivíduos distribuídos em duas famílias (Libellulidae e Gomphidae) 23 gêneros e 39 espécies (Tabela 2). Dentre os Zygoptera, as espécies Epipleoneura williansoni Santos, 1957, Hetaerina curvicauda Garrison, 1990 e Argia tinctipennis Selys, 1865 foram as mais abundantes $(\mathrm{n}=238, \mathrm{n}=185$, $\mathrm{n}=171$, respectivamente) e entre os Anisoptera, as espécies Erythrodiplax fusca (Rambur, 1842), Erythrodiplax basalis (Kirby, 1897) e Perithemis lais (Perty, 1834) ( $\mathrm{n}=64, \mathrm{n}=59$, $\mathrm{n}=54$, respectivamente).

Das 79 espécies registradas, 46 foram coletadas em córregos preservados sendo que 28 destas foram compartilhadas com córregos alterados e degradados, restando 18 espécies exclusivas de ambientes preservados. Os córregos alterados apresentaram 66 espécies, das quais 56 compartilhadas com córregos degradados (Tabela 2).

\section{Riqueza de espécies nos córregos estudados}

Quando analisadas as riquezas estimadas para Odonata por pontos de amostragem, as maiores riquezas ocorreram nos córregos RIBET1 $(59,75 \pm 4,98)$ (média \pm intervalo de confiança), RIBET2 $(35,88 \pm 2,47)$ e CRSRI $(26,93 \pm 1,92)$ 
Tabela 2 - Espécies coletadas em cada uma das três categorias de conservação nos pontos amostrados na Bacia Hidrográfica do Rio Suiá-Missu, MT, Brasil.

\begin{tabular}{|c|c|c|c|}
\hline Espécies & Alterado & Degradado & Preservado \\
\hline Acanthagrion cuyabe Calvert, 1909 & 0 & 0 & 23 \\
\hline Acanthagrion jessei Leonard, 1977 & 0 & 1 & 0 \\
\hline Acanthagrion minutum Leonard, 1977 & 0 & 8 & 10 \\
\hline Acanthagrion phallicorne Leonard, 1977 & 1 & 24 & 8 \\
\hline Acanthagrion truncatum Selys, 1876 & 33 & 8 & 41 \\
\hline Agriogomphus sp. & 0 & 0 & 2 \\
\hline Anatya guttata (Erichson, 1848) & 1 & 0 & 1 \\
\hline Argia croceipennis Selys, 1865 & 0 & 0 & 5 \\
\hline Argia lilacina Selys, 1865 & 0 & 55 & 0 \\
\hline Argia reclusa Selys, 1865 & 6 & 0 & 36 \\
\hline Argia tinctipennis Selys, 1865 & 12 & 35 & 124 \\
\hline Argyrothemis argêntea Ris, 1909 & 2 & 3 & 1 \\
\hline Chalcopteryx rutilans (Rambur, 1842) & 0 & 16 & 0 \\
\hline Cyanallagma ferenigrum De Marmels, 2003 & 33 & 17 & 2 \\
\hline Dasythemis sp. & 0 & 1 & 0 \\
\hline Diastatops intensa Montgomery, 1940 & 35 & 7 & 0 \\
\hline Diastatops obscura (Fabricius, 1775) & 23 & 4 & 8 \\
\hline Dythemis sp Hagen, 1861 & 0 & 3 & 0 \\
\hline Elasmothemis cannacrioides (Calvert, 1906) & 2 & 0 & 0 \\
\hline Elga sp. & 0 & 0 & 1 \\
\hline Epipleoneura sp. nov. * & 15 & 0 & 29 \\
\hline Epipleoneura metallica Rácenis, 1955 & 9 & 8 & 95 \\
\hline Epipleoneura westfalli Machado, 1986 & 32 & 10 & 42 \\
\hline Epipleoneura williansoni Santos, 1957 & 133 & 105 & 0 \\
\hline Epipleoneura venezuelensis Rácenis, 1955 & 0 & 0 & 9 \\
\hline Erythemis credula Hagen, 1861 & 2 & 7 & 0 \\
\hline Erythrodiplax amazonica Sjöstedt, 1918 & 0 & 1 & 0 \\
\hline Erythrodiplax basalis Kirby 1897 & 13 & 46 & 0 \\
\hline Erythrodiplax sp. & 1 & 7 & 0 \\
\hline Erythrodiplax fusca ( Rambur, 1842) & 4 & 60 & 0 \\
\hline Erythrodiplax juliana Ris, 1911 & 0 & 7 & 0 \\
\hline Erythrodiplax latimaculata Ris, 1911 & 0 & 6 & 0 \\
\hline Erythrodiplax maculosa (Hagen, 1861) & 22 & 20 & 0 \\
\hline Erythrodiplax ochracea (Burmeister, 1839) & 15 & 3 & 0 \\
\hline Erythrodiplax paraguayensis (Förster, 1904) & 1 & 13 & 0 \\
\hline Erythrodiplax umbrata (Linnaeus, 1758) & 0 & 1 & 0 \\
\hline Fylgia amazonica Kirby, 1889 & 0 & 12 & 5 \\
\hline Gen. nov., sp. nov. 1 * & 29 & 75 & 21 \\
\hline Gen.nov., sp.nov. 2 * & 69 & 14 & 6 \\
\hline Heliocharis amazona Selys, 1853 & 35 & 0 & 8 \\
\hline Helveciagrion obsoletum (Selys, 1876) & 8 & 53 & 0 \\
\hline Hetaerina curvicauda Garrison, 1990 & 97 & 48 & 40 \\
\hline Hetaerina rosea Selys, 1853 & 0 & 0 & 1 \\
\hline Hetaerina westfalli Rácenis, 1968 & 5 & 0 & 49 \\
\hline Heteragrion icterops Selys, 1862 & 0 & 0 & 2 \\
\hline Idiataphe amazonica (Kirby, 1889) & 0 & 6 & 0 \\
\hline Ischnura capreolus (Hagen, 1861) & 6 & 29 & 1 \\
\hline Macrothemis imitans Karsch, 1890 & 0 & 0 & 1 \\
\hline Miathyria marcella (Selys, 1857) & 0 & 1 & 0 \\
\hline Miathyria simplex (Rambur, 1842) & 0 & 3 & 0 \\
\hline Micrathyria eximia Kirby, 1887 & 0 & 30 & 0 \\
\hline
\end{tabular}


Tabela 2 - Continuação

\begin{tabular}{|c|c|c|c|}
\hline Espécies & Alterado & Degradado & Preservado \\
\hline Mnesarete aenea (Selys, 1853) & 0 & 0 & 17 \\
\hline Mnesarete machadoi Garrison, 2006 & 0 & 0 & 1 \\
\hline Neoneura denticulata Williamson, 1917 & 0 & 0 & 1 \\
\hline Neoneura gaida Rácenis, 1953 & 3 & 0 & 0 \\
\hline Neoneura lucas Machado, 2002 & 19 & 0 & 0 \\
\hline Neoneura luzmarina De Marmels, 1989 & 8 & 3 & 8 \\
\hline Neoneura sylvatica Hagen, 1886 & 1 & 0 & 4 \\
\hline Oligoclada abreviata (Rambur, 1842) & 1 & 0 & 0 \\
\hline Oligoclada amphinome Ris, 1919 & 0 & 1 & 0 \\
\hline Oligoclada xanthopleura Borror, 1931 & 5 & 1 & 7 \\
\hline Oligoclada walkeri Geijskes, 1931 & 0 & 0 & 2 \\
\hline Orthemis discolor (Burmeister, 1839) & 0 & 4 & 0 \\
\hline Perithemis lais (Perty, 1834) & 14 & 16 & 24 \\
\hline Perithemis mooma Kirby, 1889 & 0 & 4 & 0 \\
\hline Phasmoneura exigua (Selys, 1886) & 0 & 6 & 1 \\
\hline Phasmoneura janirae Lencioni, 1999 & 0 & 0 & 1 \\
\hline Phyllocycla armata Belle, 1977 & 0 & 2 & 0 \\
\hline Phoenicagrion sp. nov. * & 18 & 0 & 1 \\
\hline Planiplax phoenicura Ris, 1912 & 5 & 1 & 0 \\
\hline Progomphus sp. & 0 & 2 & 0 \\
\hline Oxiagrion fernandoi Costa 1988 & 2 & 0 & 3 \\
\hline Telebasis carminita Calvert, 1909 & 2 & 1 & 1 \\
\hline Telebasis coccinea (Selys, 1876) & 4 & 18 & 5 \\
\hline Telebasis racenisi Bick \& Bick, 1995 & 21 & 11 & 0 \\
\hline Tholymis citrina Hagen, 1867 & 0 & 0 & 1 \\
\hline Tigriagrion auratinigrum Calvert, 1909 & 0 & 3 & 0 \\
\hline Zenitoptera fasciata (Linnaeus, 1758) & 6 & 9 & 0 \\
\hline Zenithoptera viola Ris, 1910 & 3 & 0 & 0 \\
\hline
\end{tabular}

*sp. nov. = espécie nova; Gen. nov., sp. nov. = gênero novo, espécie nova.

(Figura 3A). Analisando por subordem, Anisoptera teve a maior riqueza estimada nos córregos RIBET2 $(23,92 \pm 2,12)$, RIBET1 $(18,90 \pm 3,04)$ e CRSRI $(12,95 \pm 1,67)$ (Figura 3B); e Zygoptera foi maior nos córregos RISUZ $(21,92 \pm 5,08)$, $\operatorname{CRL}(19,90 \pm 2,30)$ e RIBET2 $(17,92 \pm 2,12)$ (Figura 3C).

As maiores riquezas estimadas para a ordem Odonata, por níveis de conservação dos córregos, ocorreu em pontos classificados como degradados $(60,95 \pm 5,76)$ (Figura 4A), apresentando em média 11 espécies a mais que áreas alteradas e 14 a mais que áreas preservadas. Quando comparadas as riquezas entre áreas preservadas e alteradas, não houve diferença significativa.

Analisando por subordem, Anisoptera diferiu quanto a riqueza estimada em todos os níveis de conservação, com a maior riqueza estimada nos córregos classificados como degradados $(36,96 \pm 5,11)$, apresentando em média 15 espécies a mais que áreas alteradas e 24 a mais do que áreas preservadas. Por sua vez, ambientes alterados apresentaram em média nove espécies a mais do que ambientes preservados (Figura 4B).
Para a subordem Zygoptera, a maior riqueza foi observada em córregos considerados como preservados $(34,25 \pm 5,13)$, apresentando em média seis espécies a mais que alterados e 10 a mais que degradados. Resultado similar foi obtido quando comparado a riqueza de ambientes alterados com degradados, locais alterados apresentaram em média quatro espécies a mais (Figura 4C).

\section{Composição de Odonata em diferentes níveis de conservação}

Com base na similaridade de composição de Odonata existe uma separação entre os locais preservados diferindo-se de alterados e degradados (Figura 5A). No entanto, existe uma sobreposição de córregos pertencentes às categorias alterados e degradados. $\mathrm{O}$ mesmo foi corroborado pela análise de similaridade (ANOSIM: $r=0,467 ; \mathrm{p}=0,011$ ). Quando a comparação foi realizada par-a-par, os córregos preservados e alterados diferiram quanto à similaridade da composição de Odonata (ANOSIM: $r=0,519 ; \mathrm{p}=0,032$ ), sendo a mesma diferença observada quando comparados córregos preservados e degradados (ANOSIM: $r=0,785 ; \mathrm{p}=0,013$ ). Porém não houve diferença de composição entre córregos alterados 

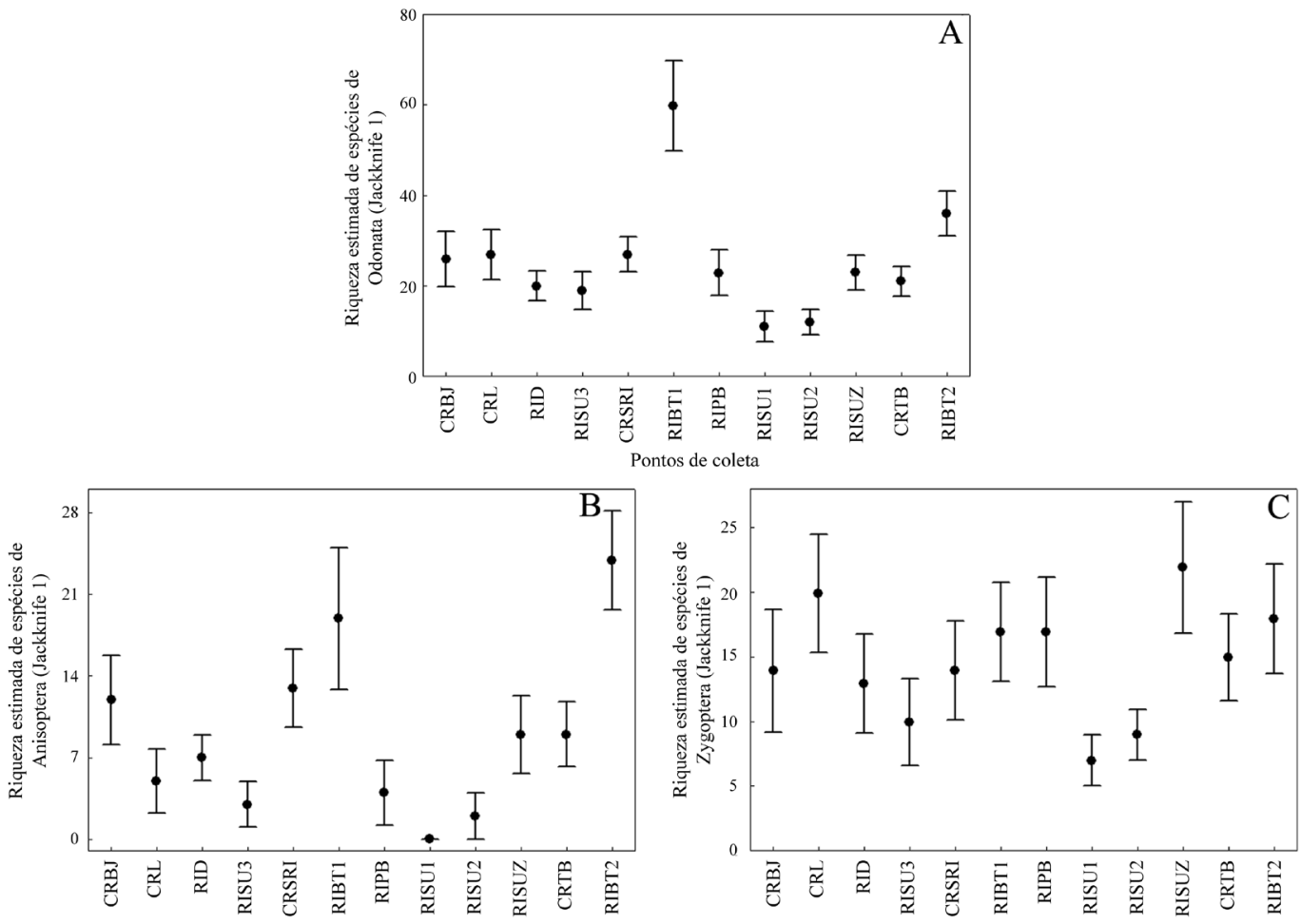

Figura 3 -Riqueza estimada de espécies (jackknife de primeira ordem) por ambientes amostrados da Bacia do Rio Suiá-Missu, MT, Brasil: A) para ordem Odonata; B) para subordem Anisoptera; C) para subordem Zygoptera. As barras representam o intervalo de confiança de 95\%.
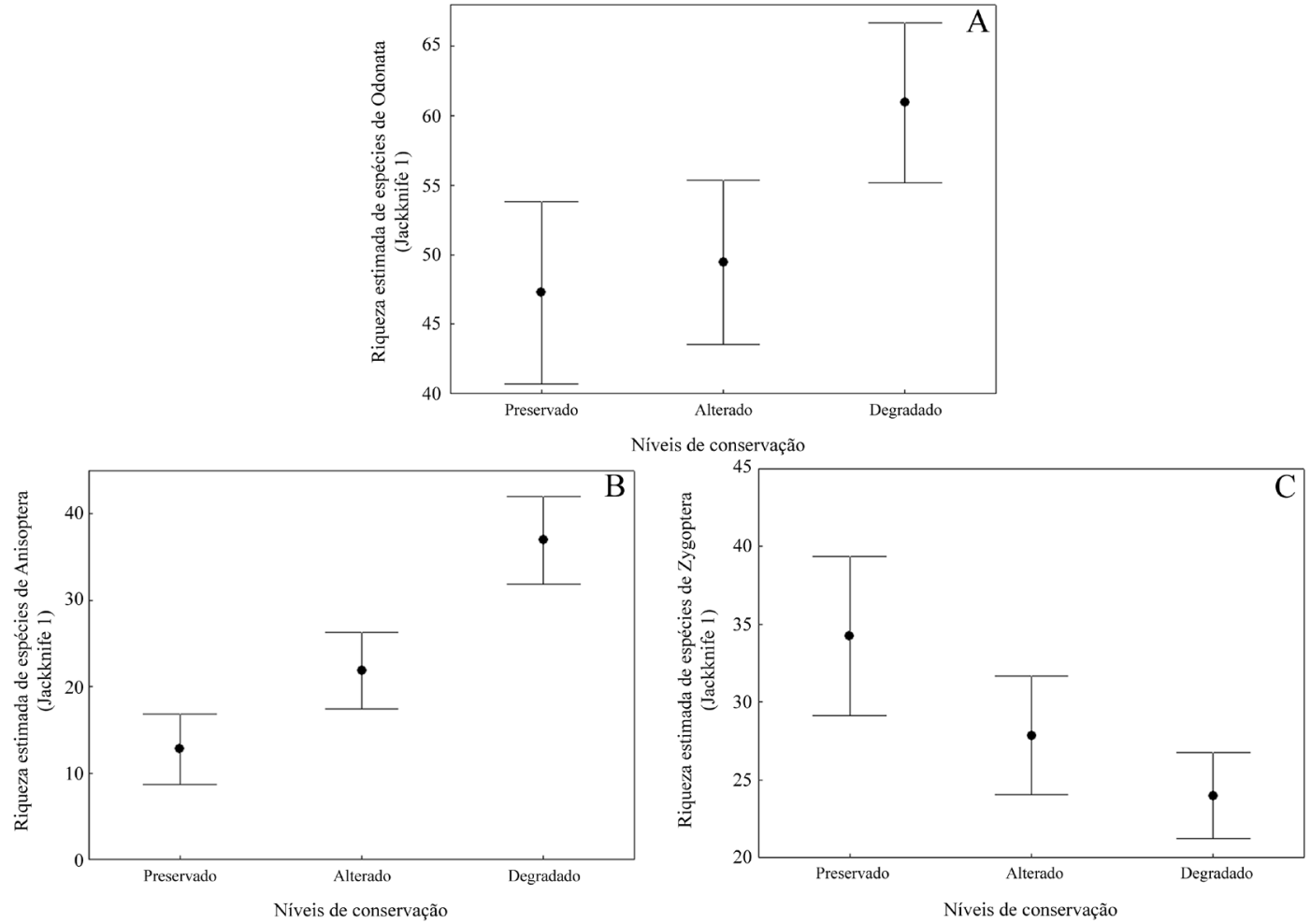

Figura 4 -Riqueza estimada de espécies (jackknife de primeira ordem) por níveis de conservação dos ambientes amostrados da Bacia do Rio Suiá-Missu, MT, Brasil: A) para ordem Odonata; B) para subordem Anisoptera; C) para subordem Zygoptera. As barras representam 0 intervalo de confiança de $95 \%$. 
e degradados (ANOSIM: $\mathrm{r}=-0,074 ; \mathrm{p}=0,518$ ). Avaliando separadamente, para Anisoptera, existe uma distinção apenas entre locais preservados e alterados/degradados, sendo que estes últimos ficaram sobrepostos. Quando a análise de similaridade foi feita comparando os três tratamentos, não houve diferença (ANOSIM: $\mathrm{r}=0,195 ; \mathrm{p}=0,073$ ) (Figura 5B). No entanto, houve diferença na similaridade apenas quando comparados córregos preservados e alterados (ANOSIM: $r=0,287 ; p=0,035$ ), e não houve diferença na similaridade quando comparados córregos preservados e degradados (ANOSIM: $\mathrm{r}=0,220 ; \mathrm{p}=0,099)$ e córregos alterados e degradados (ANOSIM: r=0,083; $\mathrm{p}=0,281$ ) (Figura 5B).

Para a subordem Zygoptera pode-se observar que existe uma separação clara entre córregos preservados e degradados, enquanto os córregos alterados se encontraram dispersos no gráfico (ANOSIM: $r=0,451 ; p=0,012$ ) (Figura 5 C). Porém, a análise de similaridade detectou diferenças também entre as categorias preservadas e alteradas (ANOSIM: $r=0,519$; $\mathrm{p}=0,025)$, bem como, entre preservados e degradados (ANOSIM: $r=0,774 ; \mathrm{p}=0,023$ ), mas não houve diferença entre córregos alterados e degradados (ANOSIM: $r=0,037$; $\mathrm{p}=0,375)$ (Figura 5C).

\section{DISCUSSÃO}

A grande variação na riqueza e composição das comunidades de Odonata nos diferentes níveis de conservação dos igarapés da Bacia do Suiá-Missu pode estar diretamente relacionada às alteraçóes na mata ciliar que, segundo Voelz e McArthur (2000), podem refletir diretamente na estruturação das comunidades. Em grande parte, os igarapés classificados como degradados foram os ambientes com maior riqueza estimada de espécies de Odonata, sendo observado o mesmo padrão quando analisada a subordem Anisoptera. Uma vez que o estimador de riqueza utilizado leva em consideração a abundância dos organismos estudados (pois considera como espécie rara aquela que ocorreu apenas uma única vez no estudo, o que eleva o número de espécies estimadas, dessa forma quanto maior a raridade maior será o número de espécies estimadas), mesmo com uma riqueza similar entre Anisoptera e Zygoptera, a baixa abundância de Anisoptera ( $21 \%$ da abundância total), provavelmente, influenciou o resultado geral, levando os córregos degradados a se apresentarem mais ricos.

Nossa hipótese foi corroborada, havendo diferença na riqueza das subordens de Odonata. Os córregos degradados
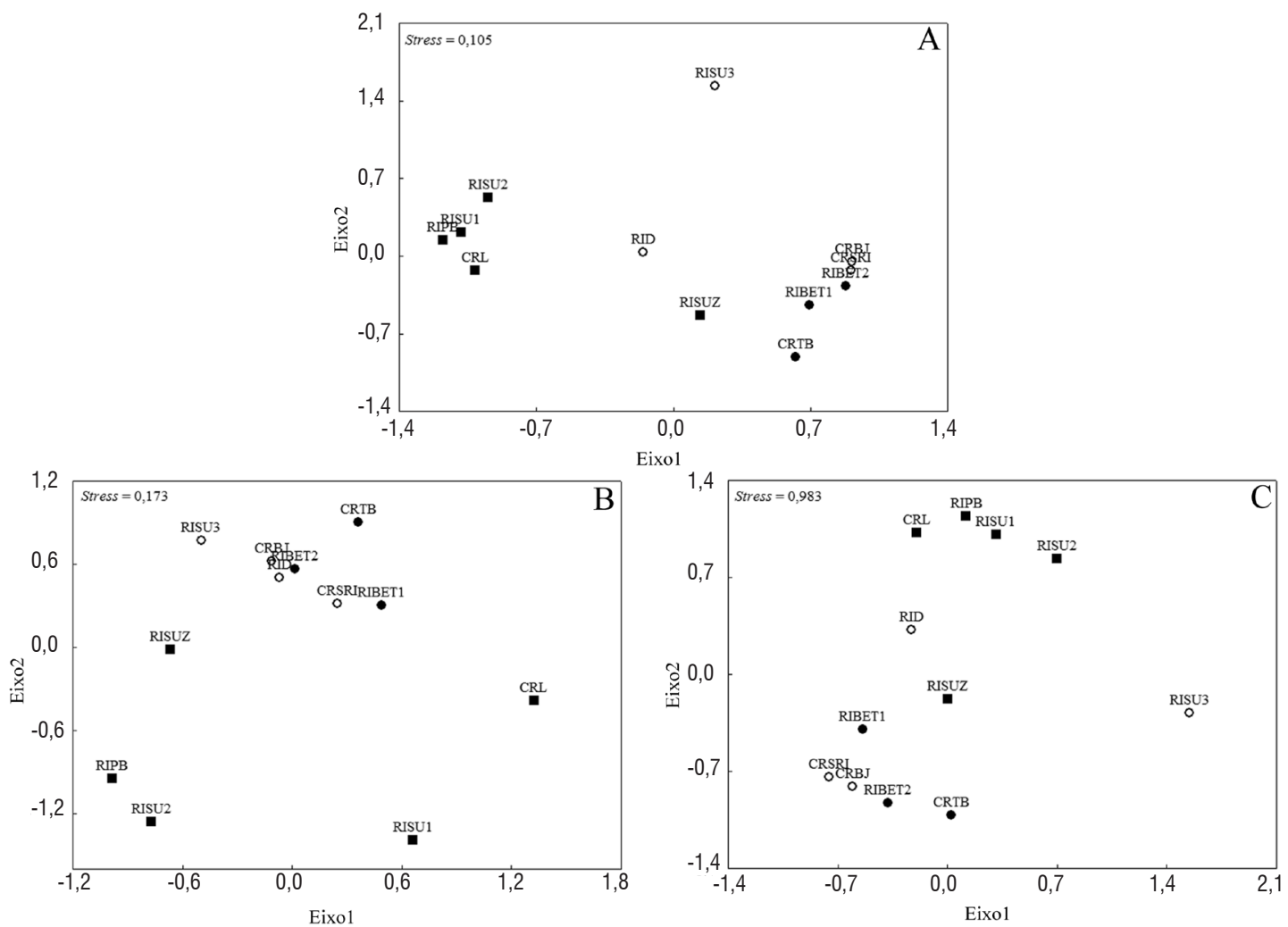

Figura 5. -Ordenação de escalonamento multidimensional não-métrico (NMDS) das categorias (preservado, alterado e degradado) dos ambientes amostrados com relação a composição de espécies da Bacia do Rio Suiá-Missu, Mato Grosso, Brasil A) da ordem Odonata; B) da subordem Anisoptera; C) da subordem Zygoptera (preservado; alterado e degradado)dos ambientes amostrados com relação a composição de espécies da Bacia do Rio Suiá-Missu, Mato Grosso, Brasil A) da ordem Odonata; B) da subordem Anisoptera; C) da subordem Zygoptera (— preservado; O alterado e $\bullet$ degradado).. 
apresentaram maior riqueza de espécies de Anisoptera do que alterados e preservados, e córregos preservados tiveram maior riqueza de espécies de Zygoptera. Uma explicação para a maior riqueza de espécies de Anisoptera em ambientes degradados é devido às alteraçóes nas matas ciliares que possibilitaram maior entrada de luz e calor nos sistemas. A alta luminosidade favorece essa subordem, pois para iniciarem suas atividades necessitam da incidência da radiação solar em seus corpos para aquecer (May 1976; Corbet e May 2008; Resende 2010). Alguns estudos ressaltam a importância da sombra e do sol na seleção de hábitat de Odonata em riachos (Calvão et al. 2013), sugerindo que adultos de muitas espécies de Anisoptera evitam as áreas sombreadas dos corpos d'água e que a abundância média diminui quando a intensidade de sombreamento aumenta (Kinvig e Samways 2000; Samways e Taylor 2004; Remsburg et al. 2008).

Além disso, a alta riqueza de espécies em ambientes degradados pode ter sido reflexo da proximidade com represas (ambiente lêntico), onde muitas destas espécies podem ter dispersado para os igarapés amostrados, possibilitando também, o estabelecimento daquelas espécies adaptadas a ambientes lênticos e de maior entrada de luz (De Marco e Latini 1998; Carvalho e Nessimian 1998).

Já a alta riqueza de espécies de Zygoptera em locais preservados, juntamente com a alta abundância amostrada nesses locais, pode ser indício de que os sistemas estão em equilíbrio. Zygoptera é um grupo de insetos que normalmente estão associados a locais mais íntegros com vegetação ribeirinha conservada devido às necessidades biológicas mais especificas (Corbet 1999). Em geral possuem um menor tamanho corporal e mostram elevada condutância (alta troca de calor corporal com a temperatura do ambiente) e a temperatura do corpo varia com a do ambiente. Por isso, devido as suas restriçóes ecofisiológicas ficariam mais associadas a ambientes florestados (May 1976; Cobert 1999). Em geral as espécies presentes em riachos de cabeceira, em florestas tropicais, tendem a ser de pequeno tamanho corporal e, consequentemente, com maior dependência da temperatura local (que varia pouco dentro de florestas maduras), e ainda, possuem baixa capacidade de dispersão (Juen e De Marco 2011). Além disso, a presença de vegetação aquática aumenta a complexidade dos ambientes e isso pode ser um dos fatores preponderantes para o sucesso das larvas desse taxa e beneficiando-as, uma vez que, em sua maioria apresentam comportamento escalador e agarrador com ontogenia associada à vegetação (Corbet, 1999; Juen e De Marco 2007).

A hipótese de que os córregos alterados e degradados se comportariam diferente de preservados com relação a composição de espécies foi corroborada quando analisado a ordem Odonata como um todo e com as duas subordens separadamente. Este fato indica, mais uma vez, a importância da mata ciliar e sua conservação para a fauna de Odonata. A composição de espécies manteve-se distinta entre córregos conservados e córregos alterados e degradados, uma vez que espécies com exigências ambientais mais específicas, com amplitude de nicho mais restrito quanto à variação de temperatura e incidência solar, ficariam mais associadas a córregos com maior quantidade de vegetação. Já as espécies com menores restriçôes ambientais poderiam habitar ambientes mais abertos. Conclusôes similares foram feitas por Monteiro-Júnior et al. (2013) e Carvalho et al. (2013).

Alguns estudos demonstram que muitas vezes a retirada da vegetação ripária expóe esses ambientes a luz solar, favorecendo o crescimento vegetal rápido, especialmente de gramíneas, fornecendo, assim, hábitats adequados para determinadas espécies (Samways e Sharratt 2009). Este cenário (mistura de vegetação, disponibilidade de sol e sombra) pode tamponar o efeito da perturbação, e os novos ambientes, criados pela ação de agentes perturbadores, parecem ser favoráveis a muitas espécies de Odonata (Samways e Steytler 1996; Stewart e Samways 1998; Carvalho et al. 2013), sendo essa assertiva corroborada por Paulson (2006) ao descrever a importância das florestas para libélulas Neotropicais.

\section{CONCLUSÃO}

O padrão de riqueza e composição de adultos de Odonata esteve fortemente relacionado com o nível de conservação dos córregos estudados. Conforme o esperado, córregos alterados e degradados comportaram uma composição de espécies diferente dos preservados. Zygoptera que geralmente habita áreas com cobertura vegetal densa, foi realmente mais rico em ambientes preservados, enquanto que Anisoptera foi mais rico em áreas com menor cobertura, sendo favorecido pela maior abertura de dossel.

\section{AGRADECIMENTOS}

Ao laboratório de Entomologia da Universidade do Estado de Mato Grosso-UNEMAT pelo apoio institucional e acadêmico, à Coordenação de Aperfeiçoamento de Pessoal de Nível Superior-CAPES e Fundação Amazônia Paraense pela concessão de bolsa de estudos. Ao Conselho Nacional de Desenvolvimento Científico e Tecnológico-CNPq pelo fomento (proc. no 520268/2005-9). Ao especialista Frederico A. A. Lencioni pelo auxilio na identificaçáo das espécies de Zygoptera. A Denis Silva Nogueira, Núbia Franças da Silva Giehl, Vanessa Depra, Karina Dias Silva e Herson Lima pelo auxilio na coleta de dados. A Empresa Brasileira de Pesquisa Agropecuária - EMBRAPA - Meio Ambiente, Instituto Sócio Ambiental - ISA e ONGs relacionadas à Campanha Y Ikatu Xingu, incentivadores deste projeto. 


\section{BIBLIOGRAFIA CITADA}

Alves-Martins, F.; Del-Claro, K.; Jacobucci, G.B. 2012. Sexual size dimorphism, mating system and seasonality of a Neotropical damselfly, Telebasis carmesina (Coenagrionidae). International Journal of Odonatology, 15: 263-273.

Bojsen, B.H.; Jacobsen, D. 2003. Effects of deforestation on macroinvertebrate diversity and assemblage structure in Ecuadorian Amazonian. Archiv für Hydrobiologie, 158: 317-342.

Callisto, M.; Moretti, M.; Goulart, M. 2001. Macroinvertebrados bentônicos como ferramenta para avaliar a saúde de riachos. Revista Brasileira de Recursos Hidricos, 6: 71-82.

Calvão, L.B.; Vital, M.V.C.; Juen, L.; Lima Filho, G.F.; Oliveira-Junior, J.M.B.; Pinto, N.S.; De Marco, Jr. P. 2013. Thermoregulation and microhabitat choice in Erythrodiplax latimaculata Ris males (Anisoptera: Libellulidae). Odonatologica, 42: 97-108.

Carvalho, A.L.; Nessimian, J.L. 1998. Odonata do Estado do Rio de Janeiro, Brasil: Hábitats e hábitos das larvas, p. 3-28. In: Nessimian, J.L.; Carvalho, A.L. (Eds.). Ecologia de Insetos Aquáticos. Rio de Janeiro. Séries Oecologia Brasiliensis PPGEUFRJ, 309p.

Carvalho, F.G.; Silva-Pinto, N.; Oliveira-Júnior, J.M.B.; Juen, L. 2013. Effects of marginal vegetation removal on Odonata communities. Acta Limnologica Brasiliensia, http://dx.doi. org/10.1590/S2179-975X2013005000013.

Clarke, K.R.; Warwick, R.M.. 1994. Chance in marine communities: an approach to statistical analysis and interpretation. Bourne Press, Bournemouth, UK, 128p.

Colwell, R.K. 2005. EstimateS: Statistical estimation of species richness and shared species from samples. Version 7.5. (http:// purl.oclc.org/estimates). Acesso em 17/03/2012.

Colwell, R.K.; Coddington, J.A. 1994. Estimating terrestrial biodiversity through extrapolation. Philosophical Transactions of the Royal Society of London, 345: 101-118.

Corbet, P.S. 1999. Dragonflies: Behavior and Ecology of Odonata. Comstock Publ. Assoc., Ithaca, NY, 829p.

Corbet, P.S.; May, M.L. 2008. Fliers and perchers among Odonata: dichotomy or multidimensional continuum? A provisional reappraisal. International Journal of Odonatology, 11: 155-171.

De Marco, P.Jr.; Latini, A.O. 1998. Estrutura de guildas e riqueza de espécies em uma comunidades de larvas de Anisoptera (Odonata), p.101-112. In: Nessimian, J.L.; Carvalho A.L. (Eds). Ecologia de insetos aquáticos. Séries Oecologia Brasiliensis, PPGE-UFRJ, Rio de Janeiro, RJ, 309p.

De Marco, P.Jr.; Resende, D.C. 2002. Activity patterns and thermoregulation in a tropical dragonfly assemblage. Odonatologica, 31: 129-138.

Dias-Silva, K; Cabette H.R.S.; Juen, L.; De Marco, P.J. 2010. The influence of habitat integrity and physical-chemical water variables on the structure of aquatic and semi-aquatic Heteroptera. Zoologia, 27: 918-930.

Garrison, R.W.; Von Ellenrieder, N.; Louton, J.A. 2006. Dragonfly genera of the new word: an illustrated and annotated key to the Anisoptera. The Johns Hopkins University Press, Baltimore, MD, 368p.
Garrison, R.W.; Von Ellenrieder, N.; Louton, J.A. 2010. Damselfly genera of the New World: an Illustrated and annotated key to the Zygoptera. The Johns Hopkins University Press, Baltimore, MD, 490p.

Guillermo-Ferreira, R.; Del-Claro, K. 2012. Reproductive behavior of Acanthagrion truncatum Selys, 1876 (Odonata: Coenagrionidae). International Journal of Odonatology, 15: 299-304.

Heckman, C. 2008 . Encyclopedia of South American aquatic insects: Odonata - Zygoptera. Springer Science, Olympia, WA, 692p.

Heiser, M.; Schmitt, T. 2010. Do different dispersal capacities influence the biogeography of the western Palearctic dragonflies (Odonata)? Biological Journal of the Linnean Society, 99: 177-195.

Juen, L.; Cabette, H.S.R.; De Marco, P.Jr. 2007. Odonate assemblage structure in relation to basin and aquatic habitat structure in Pantanal wetlands. Hydrobiologia, 579: 125-134.

Juen, L.; De Marco, P.Jr. 2011. Odonate beta diversity in terra-firme forest streams in Central Amazonia: On the relative effects of neutral and niche drivers at small geographical extents. Insect Conservation and Diversity, 4: 265-274.

Juen L.; De Marco P. Jr. 2012. Dragonfly endemism in the Brazilian Amazon: competing hypotheses for biogeographical patterns. Biodiversity and Conservation, 21: 3507-3521.

Kinvig, R.G.; Samways, M.J. 2000. Conserving dragonflies (Odonata) along streams running through commercial forestry. Odonatologica, 29: 195-208.

Legendre, P.; Legendre, L. 1998. Numerical Ecology. 2. ed. Elsevier, Amsterdam. 853p.

Lencioni, F.A.A. 2005. The damselflies of Brazil: an illustrated guideThe non Coenagrionidae families. All Print Editora. 332p.

Lencioni, F.A.A. 2006. The damselflies of Brazil: an illustrated guide Coenagrionidae. All Print Editora, São Paulo,SP, 743p.

Maeda, E.E.; Formaggio, A.R.; Shimabukuro, Y.E. 2008. Análise histórica das transformaçóes da Floresta Amazônica em áreas agrícolas na Bacia do Rio Suiá-Missu. Sociedade e Natureza, 20: 5-24.

May, M.L. 1976. Thermoregulation in adaptation to temperature in dragonflies (Odonata: Anisoptera). Ecological Monographs, 46: 1-32.

Monteiro-Júnior, C.S.; Couceiro, S.R.M.; Hamada, N.; Juen, L. 2013. Effect of vegetation removal for road building on richness and composition of Odonata communities in Amazonia, Brazil, International Journal of Odonatology, 17:1-13.

Nessimian, J.L.; Venticinque, E.; Zuanon, J.; De Marco, P.Jr.; Gordo, M.; Fidelis, L.; Batista, J.D.; Juen, L. 2008. Land use, habitat integrity, and aquatic insect assemblages in Central Amazonian streams. Hydrobiologia, 614: 117-131.

Oliveira-Júnior J.M.B.; Cabette H.S.R.; Pinto N.S.; Juen, L. 2013. As variaçóes na comunidade de Odonata (Insecta) em córregos podem ser preditas pelo Paradoxo do Plâncton? Explicando a riqueza de espécies pela variabilidade ambiental. EntomoBrasilis, 6: $1-8$. 
Paulson, D. 2006. The importance of forest to Neotropical Dragonflies. Forest and Dragonflies Fourth WDA International Symposium of Odonatology Pontevedra Spain: 79-101.

Pereira, L.R.; Cabette, H.S.R.; Juen, L. 2012. Trichoptera as bioindicators of habitat integrity in the Pindaíba river basin, Mato Grosso (Central Brazil). Annales of Limnologie, 48: 295302.

R Development Core Team. 2011. R: a language and environment for statistical computing. R Foundation for Statistical Computing, Vienna, Austria. ISBN 3-900051-07-0 (http://www.R-project. org/). Acesso em 20/11/2012.

Ratter, J.A.; Askew, G.P.; Montgomery, R.F.; Gifford, D.R. 1978. Observations on vegetation of Northeastern Mato-Grosso. 2. Forests and soils of Rio Suia-Missu area. Proceedings of the Royal Society of London, Series B-Biological Sciences, 203: 191-208.

Remsburg, A.J.; Olson, A.C.; Samways, M.J. 2008. Shade alone reduces adult dragonfly (Odonata: Libellulidae) abundance. Journal Insect Behaviour, 21: 460-468.

Resende, D.C. 2010. Residence advantage in heterospecific territorial disputes of Erythrodiplax Brauer species (Odonata, Libellulidae). Revista Brasileira de Entomologia, 54: 110-114.

Samways, M.J.; Steytler, N.S. 1996. Dragonfly (Odonata) distribution patterns in urban and forest landscapes, and recommendations for riparian management. Biological Conservation, 78: 279-288.
Samways, M.J.; Sharratt, N.J. 2009. Recovery of endemic dragonflies after removal of invasive alien trees. Conservation Biology, 24: 267-277.

Samways, M.J.; Taylor, S. 2004. Impacts of invasive alien plants on Red-Listed South African dragonflies (Odonata). South African Journal of Science, 100: 78-80.

Silva, D.P.; De Marco, P.Jr,; Resende, D.C. 2010. Adult Odonate abundance and community assemblage measures as indicators of stream ecological integrity: A case study. Ecological Indicators, 10: 744-752.

Silva-Pinto, N; Juen, L.; Cabette, H.S.R.; De Marco, P. Jr. 2012. Fluctuating asymmetry and wing size of Argia tinctipennis Selys (Zygoptera: Coenagrionidae) in relation to Riparian Forest Preservation Status. Neotropical Entomology, 41: 1-9.

Stewart, D.A.B.; Samways, M.J. 1998. Conserving dragonfly (Odonata) assemblages relative to river dynamics in an African savanna game reserve. Conservation Biology, 12: 683-692.

Strahler, H.N. 1957. Quantitative analysis of watershed geomorphology. American Geophysical Union Transactions, 38: 913-920.

Voelz, N.J.; McArthur, V. 2000. An exploration of factors influencing lotic insect species richness. Biodiversity and Conservation, 9: 1543-1570.

Recebido em 01/04/2013

Aceito em 22/07/2013 
